\title{
A regulatory SNP in AKAP13 is associated with blood pressure in Koreans
}

\author{
Kyung-Won Hong, Ji-Eun Lim and Bermseok Oh
}

High blood pressure contributes to more than 10 million deaths per year worldwide through stroke and ischemic heart disease. Yet, genome-wide association studies (GWASs) have identified a small fraction of its underlying genetic factors. To identify biologically important single-nucleotide polymorphisms (SNPs) that regulate variations in blood pressure, we analyzed SNPs in a genome-wide association study. Genome-wide genotype data (original study $n=7551, \mathrm{SNP}=352228$; replication study $n=3703$, $\mathrm{SNP}=20$ ) were obtained from the Korea National Institute of Health, wherein 29921 of 352228 SNPs lay within 5 kbp upstream of genes. Linear regression analysis was performed for systolic and diastolic blood pressure (DBP) by controlling for cohort, age, sex and body mass index. For the 20 SNPs that were associated with both blood pressure values, a replication study was performed in an independent population. A total of 20 SNPs were significantly associated with both blood pressure values in the original study, 13 of which lay in a conserved transcription factor-binding site. One SNP (rs11638762), in the GATA-3 binding site upstream of the AKAP13 gene, was significantly replicated in another cohort ( $P$-value of the meta-analysis $=1.4 \times 10^{-5}$ for systolic blood pressure and $6.3 \times 10^{-4}$ for DBP). A functional GWAS was performed using upstream SNPs, and a novel genetic factor (AKAP13), which is essential for cardiac myocyte development in mice, was identified as a regulator of blood pressure.

Journal of Human Genetics (2011) 56, 205-210; doi:10.1038/jhg.2010.167; published online 13 January 2011

Keywords: AKAP13; blood pressure; genome-wide association study; regulatory SNP

\section{INTRODUCTION}

Blood pressure is an important clinical parameter of health. High blood pressure contributes to more than 10 million deaths per year worldwide through stroke and ischemic heart disease. ${ }^{1,2}$ It is believed that $30-50 \%$ of the variability in blood pressure is influenced by multiple genetic factors, prompting extensive efforts to identify its genetic underpinnings.

As the seminal genome-wide association study (GWAS) was reported by the Wellcome Trust Case-Control Consortium, ${ }^{3}$ such studies have identified $>250$ genetic loci, in which common genetic variants have been reproducibly associated with polygenetic traits. ${ }^{4}$ Although the loci that are responsible for common diseases and traits, such as type 2 diabetes, coronary artery disease and serum lipid levels, have been faithfully mapped by several GWASs, other diseases, such as hypertension and stroke, have been intractable to reliable GWAS mapping. ${ }^{5}$

Recently, two large meta-analyses of blood pressure GWASs were conducted in subjects of European descent (Global BPgen consortium, 34433 subjects; ${ }^{6}$ CHARGE consortium, 29136 subjects ${ }^{7}$ ). Yet, the genetic variants that have been identified by the many large blood pressure genetic studies explain merely $1 \%$ of blood pressure variation. ${ }^{7}$ Therefore, our understanding of the allelic variation that affects blood pressure in the general population is in its infancy. More diverse strategies for GWASs have been required to identify additional alleles that are associated with blood pressure.

We reported a GWAS of quantitative traits, including blood pressure, in Korean Association REsource, ${ }^{8}$ implicating the ATP2B1 gene as influencing blood pressure. Our conventional approach, however, failed to identify a sufficient number of genetic factors to understand the underlying mechanism of blood pressure regulation. Therefore, we employed an alternative approach, using nonsynonymous single-nucleotide polymorphisms (SNPs) in the GWAS data, and identified more SNPs located in arginine decarboxylase, phospholipase D1, brain-derived nuclear factor and an unknown gene (AC089987). ${ }^{9}$ Although these nonsynonymous SNPs did not have strong $P$-values $\left(1.0 \times 10^{-3}<P<4.8 \times 10^{-2}\right)$, they appeared to be biologically linked to blood pressure. ${ }^{9}$

This limited success encouraged us to examine other regulatory SNPs that were obtained in the GWAS data of Korean Association REsource, generated by Affymetrix 5.0 SNP array (Affymetrix, Inc., Santa Clara, CA, USA), to identify additional genetic factors that regulate blood pressure. Single-nucleotide changes in regulatory regions can produce new regulatory elements that are recognized by alternate transcription factors, ${ }^{10}$ and many functional analyses of regulatory SNPs have demonstrated this effect on gene expression experimentally. ${ }^{11-14}$ To this end, 29921 SNPs in the upstream region 
of genes genome-wide were analyzed for their association with blood pressure.

\section{MATERIALS AND METHODS}

\section{Subjects}

The subjects and their genotypes in the original study have been reported. ${ }^{8}$ In total, 7551 individuals came from KyungGi-Do province, near Seoul, Korea, and entered a GWAS of the link between blood pressure and upstream SNP genotypes. To recapitulate the association results, 3703 persons were recruited from community health care centers in Seoul and Busan, Korea. This study was approved by the International Review Board of the Korea National Institute of Health.

The basic characteristics and blood pressures of the subjects are listed in Table 1. In the original study, blood pressure was measured three times in the supine position. Before the first measurement, the participants rested for $5 \mathrm{~min}$, and three measurements were taken at least $30 \mathrm{~s}$ apart. The average of the three measurements was used for this study. In the replication study, blood pressure was measured twice in the seated position, and the average value was used.

\section{Genotypes}

DNA samples for the original study were isolated from the peripheral blood of participants and genotyped using the Affymetrix Genomewide Human SNP array 5.0 (Affymetrix, Inc.). In the replication study, DNA samples were genotyped using the Affymetrix Genomewide Human SNP array 6.0 (Affymetrix, Inc.). The quality controls have been described in elsewhere. ${ }^{8}$ Briefly, the accuracy of the genotyping was calculated by Bayesian robust linear modeling using the Mahalanobis distance genotyping algorithm. ${ }^{15}$

Samples that had accuracies below $98 \%$ and a high missing genotype call rate $(\geqslant 4 \%)$, high heterozygosity $(>30 \%)$ or inconsistency in sex were excluded from subsequent analyses. Individuals who had a tumor or were undergoing antihypertensive therapy were excluded. Related individuals whose estimated identity-by-state values were high $(>0.80)^{3}$ were also excluded. The 352228 genotyped SNPs (excluding sex chromosome SNPs) had a missing genotype rate below 0.1 , a minor allele frequency higher than 0.01 and no deviation from Hardy-Weinberg Equilibrium $\left(P>1 \times 10^{-6}\right)$.

\section{Upstream SNP analysis}

Using BIOMART version 5.0 (http://www.ensembl.org/biomart/), 29921 upstream SNPs were selected from the QC-filtered 352228 SNPs, based on the data in dbSNP v.130. BIOMART, a web-based tool, allowed us to use information from the latest release of a database limiting on specific upstream category.

In our quantitative analysis of systolic blood pressure (SBP) and diastolic blood pressure (DBP), linear regression was used by controlling for a subject's cohort, age, sex and body mass index.

Statistical analyses were performed using PLINK (version 1.07). ${ }^{16}$ All tests were based on an additive model and $P$-values were not adjusted for multiple tests. Significant SBP- and DBP-associated SNPs were examined in the replica-

Table 1 Basic characteristics of study subjects

\begin{tabular}{lcc}
\hline Variables & KARE & $\begin{array}{c}\text { Replication } \\
\text { samples }\end{array}$ \\
\hline $\begin{array}{l}\text { Number of individuals (\%) } \\
\text { Gender (men (\%)/women (\%)) }\end{array}$ & 7551 & 3703 \\
& $(50)$ & $1651(45) / 2052$ \\
Age (mean (M) year \pm s.d.) & $51.44 \pm 8.79$ & $53.21 \pm 8.33$ \\
Body mass index $\left(M\right.$ kg m $^{-2} \pm$ s.d.) & $24.42 \pm 3.08$ & $23.96 \pm 2.90$ \\
Systolic blood pressure (M & $115.65 \pm 17.25$ & $121.95 \pm 14.80$ \\
mm Hg \pm s.d.) & & \\
Diastolic blood pressure & $74.21 \pm 11.27$ & $77.21 \pm 10.23$ \\
$(M$ mm Hg \pm s.d.) & & \\
\hline Abbreviation: s.d., standard deviation. & &
\end{tabular}

tion study. The inverse-variance meta-analysis method, assuming fixed effects, was used to generate the meta-analysis statistics. Cochran's $\mathrm{Q}$ test was used to assess between-study heterogeneity. All meta-analysis calculations were performed using R statistical language (version 2.7.1). Transcription factor binding sites (TFBSs) were identified using TFsearch (http://molsun1.cbrc.aist.go.jp/ research/db/TFSEARCH.html), and allelic differences in TFBSs were surveyed. Transcription factor (TF) score is calculated by TF search program. It indicated the possibility of transcription factor binding on the site. The score is calculated by the web-based program. Study power was based on Korean Association REsource parameters, including minor allele frequency, effect size and mean value of the markers.

\section{RESULTS}

A total of 29921 of the 352228 genotyped SNPs lay in the upstream region $(-5 \mathrm{kbp})$ of genes genome-wide; this value is one-quarter of all upstream SNPs (131593), excluding the sex chromosomes ${ }^{10}$ (Table 2). Using these 29921 SNPs, we conducted a genome-wide association study of blood pressure (SBP and DBP). Quantile-quantile plots and genomic inflation statistics are described in Figure 1. The phenotypes had statistics that approximated $1.00(1.027 \leqslant \lambda \leqslant 1.039)$, indicating that there was no genomic inflation from population stratification in these subjects.

A total of 20 SNPs were significantly associated with both SBP and DBP $(P$-value $<0.01)$ (Table 3$)$. The study-power analyses indicated that 10 of the 20 SNPs had more than $80 \%$ study power at alpha $=0.05$, and the remaining SNPs had more than $60 \%$ study power.

Of the 20 SNPs, 12 were linked to the known functional genes (PUS7, SLC6A20, ASCL4, RNF8, BCHE, ALG2, TAAR3, FUCA2, ILDR1 and SPATS2L), and the remaining correlated with unknown genes (See Table 3). The minor alleles of 14 SNPs (rs295142, rs1900706, rs842825, rs3806650, rs11929235, rs17279465, rs195386,

Table 2 Upstream SNP distribution in each chromosome, excluding sex chromosome

Number of upstream SNPS

\begin{tabular}{|c|c|c|c|c|}
\hline \multirow[b]{2}{*}{ Chromosome } & \multicolumn{4}{|c|}{ Number of upstream SNPS } \\
\hline & $\begin{array}{l}\text { In whole genome } \\
\text { (WU et al., 2009) }\end{array}$ & $\%$ & In KARE dataset & $\%$ \\
\hline 1 & 14221 & 10.8 & 3671 & 12.3 \\
\hline 2 & 8031 & 6.1 & 3105 & 10.4 \\
\hline 3 & 5586 & 4.2 & 2438 & 8.1 \\
\hline 4 & 5829 & 4.4 & 829 & 2.8 \\
\hline 5 & 5195 & 3.9 & 1016 & 3.4 \\
\hline 6 & 8409 & 6.4 & 2362 & 7.9 \\
\hline 7 & 6999 & 5.3 & 2059 & 6.9 \\
\hline 8 & 4345 & 3.3 & 829 & 2.8 \\
\hline 9 & 6699 & 5.1 & 1599 & 5.3 \\
\hline 10 & 5402 & 4.1 & 1992 & 6.7 \\
\hline 11 & 8082 & 6.1 & 1438 & 4.8 \\
\hline 12 & 5735 & 4.4 & 1092 & 3.6 \\
\hline 13 & 2129 & 1.6 & 943 & 3.2 \\
\hline 14 & 3902 & 3.0 & 721 & 2.4 \\
\hline 15 & 3580 & 2.7 & 595 & 2.0 \\
\hline 16 & 5921 & 4.5 & 596 & 2.0 \\
\hline 17 & 10227 & 7.8 & 902 & 3.0 \\
\hline 18 & 1789 & 1.4 & 434 & 1.5 \\
\hline 19 & 8842 & 6.7 & 792 & 2.6 \\
\hline 20 & 4564 & 3.5 & 1047 & 3.5 \\
\hline 21 & 1683 & 1.3 & 586 & 2.0 \\
\hline 22 & 4423 & 3.4 & 875 & 2.9 \\
\hline Total & 131593 & 100 & 29921 & 100 \\
\hline
\end{tabular}



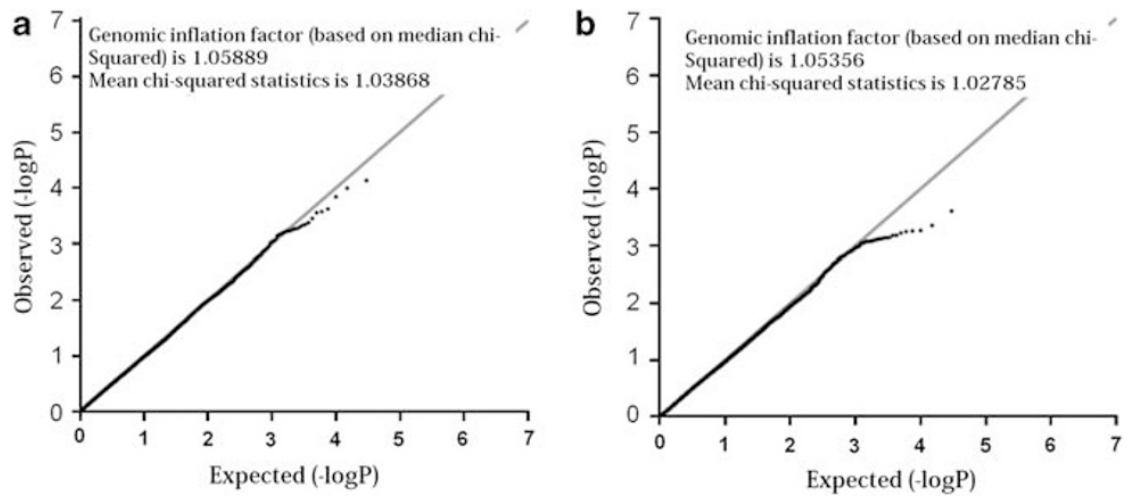

Figure 1 Quantile-quantile plot of observed and expected $P$-values for SBP (a) and DBP (b). Genomic inflation statistics are shown in the plots.

Table 3 Associated SNPs of the original genome-wide association study of blood pressure and study power for each marker

\begin{tabular}{|c|c|c|c|c|c|c|c|c|c|c|c|c|c|}
\hline \multirow[b]{2}{*}{$\mathrm{CHR}$} & \multirow[b]{2}{*}{ SNPS } & \multirow[b]{2}{*}{ Linked genes } & \multirow[b]{2}{*}{ M } & \multirow[b]{2}{*}{$M A F$} & \multirow[b]{2}{*}{ Tendency } & \multicolumn{2}{|c|}{ Systolic blood pressure } & \multicolumn{2}{|c|}{ Diastolic blood pressure } & \multirow{2}{*}{$\begin{array}{l}\text { Study } \\
\text { power }\end{array}$} & \multirow{2}{*}{$\begin{array}{l}\text { Ensemble } \\
\text { transcript ID }\end{array}$} & \multirow{2}{*}{$\begin{array}{c}\text { SNP position } \\
\text { from transcription } \\
\text { start site }\end{array}$} & \multirow{2}{*}{$\begin{array}{l}\text { Expected } \\
\text { TFBS }\end{array}$} \\
\hline & & & & & & BETA & $S B P$ & BETA & $D B P$ & & & & \\
\hline 2 & rs295142 & SPATS2L & C & 0.176 & $\downarrow$ & $-1.01 \pm 0.33$ & $2.2 \times 10^{-3}$ & $-0.73 \pm 0.22$ & $1.2 \times 10^{-3}$ & 78.3 & ENST00000409151 & -2355 & Nkx-2 \\
\hline 2 & rs1900706 & SPATS2L & $\mathrm{C}$ & 0.172 & $\downarrow$ & $-1.00 \pm 0.33$ & $2.5 \times 10^{-3}$ & $-0.73 \pm 0.22$ & $1.1 \times 10^{-3}$ & 76.7 & ENST00000409151 & -1481 & SRY \\
\hline 2 & rs842825 & SPATS2L & $\mathrm{T}$ & 0.179 & $\downarrow$ & $-0.93 \pm 0.33$ & $5.1 \times 10^{-3}$ & $-0.75 \pm 0.22$ & $7.7 \times 10^{-4}$ & 71.9 & ENST00000438761 & -2666 & \\
\hline 3 & rs3806650 & $B C H E$ & G & 0.235 & $\downarrow$ & $-0.91 \pm 0.30$ & $2.2 \times 10^{-3}$ & $-0.65 \pm 0.20$ & $1.3 \times 10^{-3}$ & 78.5 & ENSG00000114200 & -551 & AMLla \\
\hline 3 & rs11929235 & ILDR1 & A & 0.246 & $\downarrow$ & $-0.79 \pm 0.29$ & $6.6 \times 10^{-3}$ & $-0.57 \pm 0.20$ & $4.0 \times 10^{-3}$ & 67.9 & ENST00000462014 & -3901 & $\mathrm{pbx}-1$ \\
\hline 3 & rs17279465 & SLC6A2O & C & 0.047 & $\downarrow$ & $-2.00 \pm 0.60$ & $9.7 \times 10^{-4}$ & $-1.39 \pm 0.41$ & $6.8 \times 10^{-4}$ & 85.5 & ENST00000473146 & -917 & MZF1 \\
\hline 6 & rs9356478 & AL022069 & C & 0.081 & $\uparrow$ & $1.24 \pm 0.47$ & $7.8 \times 10^{-3}$ & $0.89 \pm 0.32$ & $4.9 \times 10^{-3}$ & 67.4 & ENSG00000232468 & -4716 & $\mathrm{CDP}$ \\
\hline 6 & rs3789790 & FUCA2 & G & 0.213 & $\uparrow$ & $0.91 \pm 0.31$ & $3.3 \times 10^{-3}$ & $0.62 \pm 0.21$ & $3.5 \times 10^{-3}$ & 75.7 & ENST00000451668 & -652 & S8 \\
\hline 6 & rs195386 & RNF8 & $\mathrm{T}$ & 0.113 & $\downarrow$ & $-1.52 \pm 0.41$ & $1.9 \times 10^{-4}$ & $-0.81 \pm 0.27$ & $3.3 \times 10^{-3}$ & 92.9 & ENST00000498460 & -3334 & CRE-BP \\
\hline 6 & rs6569814 & TAAR3 & $\mathrm{T}$ & 0.327 & $\uparrow$ & $0.75 \pm 0.27$ & $5.9 \times 10^{-3}$ & $0.51 \pm 0.18$ & $5.3 \times 10^{-3}$ & 70.8 & ENSG00000179073 & -1606 & Oct-1 \\
\hline 7 & rs4731112 & RP11-390E23 & C & 0.199 & $\uparrow$ & $1.05 \pm 0.32$ & $9.7 \times 10^{-4}$ & $0.62 \pm 0.22$ & $3.8 \times 10^{-3}$ & 84.8 & ENST00000422401 & -4482 & \\
\hline 7 & rs7357092 & AC006465 & $\mathrm{T}$ & 0.164 & $\uparrow$ & $1.41 \pm 0.35$ & $4.6 \times 10^{-5}$ & $0.79 \pm 0.23$ & $7.9 \times 10^{-4}$ & 96.9 & ENST00000406829 & -1870 & GATA-1 \\
\hline 7 & rs3735369 & PUS7 & $\mathrm{T}$ & 0.150 & $\downarrow$ & $-1.21 \pm 0.35$ & $6.4 \times 10^{-4}$ & $-0.82 \pm 0.24$ & $6.2 \times 10^{-4}$ & 86.8 & ENST00000487277 & -3153 & \\
\hline 8 & rs1786330 & AP001205 & $\mathrm{T}$ & 0.237 & $\downarrow$ & $-0.92 \pm 0.30$ & $2.1 \times 10^{-3}$ & $-0.58 \pm 0.20$ & $4.2 \times 10^{-3}$ & 79.6 & ENSTO0000485490 & -3586 & \\
\hline 9 & rs3780616 & $A L G 2$ & A & 0.016 & $\downarrow$ & $-2.92 \pm 1.00$ & $3.5 \times 10^{-3}$ & $-1.80 \pm 0.68$ & $7.8 \times 10^{-3}$ & 74.2 & ENSG00000119523 & -3522 & CREB \\
\hline 11 & rs10768970 & AC068205 & C & 0.240 & $\downarrow$ & $-1.04 \pm 0.30$ & $4.8 \times 10^{-4}$ & $-0.69 \pm 0.20$ & $5.5 \times 10^{-4}$ & 88.6 & ENST00000500886 & -1272 & \\
\hline 12 & rs11113470 & $A S C L 4$ & $\mathrm{G}$ & 0.451 & $\uparrow$ & $0.79 \pm 0.26$ & $2.0 \times 10^{-3}$ & $0.59 \pm 0.17$ & $6.6 \times 10^{-4}$ & 80.0 & ENST00000342331 & -3612 & \\
\hline 15 & rs11638762 & AKAP13 & $\mathrm{T}$ & 0.451 & $\downarrow$ & $-1.00 \pm 0.25$ & $9.3 \times 10^{-5}$ & $-0.47 \pm 0.17$ & $7.0 \times 10^{-3}$ & 94.4 & ENST00000452008 & -2682 & GATA-3 \\
\hline 20 & rs4811719 & AL137162 & $\mathrm{T}$ & 0.430 & $\downarrow$ & $-0.95 \pm 0.26$ & $2.4 \times 10^{-4}$ & $-0.55 \pm 0.17$ & $1.7 \times 10^{-3}$ & 91.8 & ENST00000496745 & -4653 & \\
\hline 22 & rs11089887 & $R P 3-434 P 1$ & G & 0.186 & $\downarrow$ & $-1.09 \pm 0.33$ & $8.7 \times 10^{-4}$ & $-0.70 \pm 0.22$ & $1.6 \times 10^{-3}$ & 85.6 & ENSTO0000433230 & -1617 & \\
\hline
\end{tabular}

Abbreviations: CHR, chromosome; DBP, diastolic blood pressure; M, minor allele; MAF, minor allele frequency; SBP, systolic blood pressure; SNP, single-nucleotide polymorphism; TFBS, transcription factor binding site.

aStudy power is based on KARE parameters, including minor allele frequency, effect size and mean value of the markers.

rs3735369, rs1786330, rs3780616, rs10768970, rs11638762, rs4811719 and 11089887) decreased the SBP and DBP of individuals who carried them, and the minor alleles of six SNPs (rs9356478, rs3789790, rs6569814, rs4731112, rs7357092 and rs11113470) had the opposite effect on SBP and DBP.

The 20 SNPs were examined in a replication analysis using an independent population of 3703 subjects, recruited from community health care centers in Seoul, Korea (Table 4). Their genotypes were determined using the Affymetrix Genomewide Human SNP array 6.0; 2 of the 20 SNPs were excluded during quality control. The linked gene functions and disease relations are shown in Table 5.

Three SNPs had a $P$-value $<0.05$ in the replication study. The rs3806650 revealed a significant association to DBP, but the direction of effect (beta $=0.55 \pm$ (s.e.) 0.27 ) was opposite to the original GWAS result (beta $=-0.65 \pm 0.20$ ). The rs3780616 revealed a significant association to SBP, and the direction of effect was same to the original GWAS. Meta-analysis improved the $P$-value from $3.5 \times 10^{-3}$ in GWAS to $2.0 \times 10^{-5}$. The rs11638762 had a consistent association with both SBP and DBP in the original and replication studies. In our meta-analysis, the size of the effect of rs11638762 was beta $=-0.89 \pm 0.20\left(P=1.3 \times 10^{-5}\right)$ for SBP and beta $=-0.48 \pm 0.14$ $\left(P=6.3 \times 10^{-4}\right)$ for DBP

\section{DISCUSSION}

In this study, we identified 20 upstream SNPs that significantly associated with both SBP and DBP, and three SNPs were replicated in the other cohort. As the replication samples were recruited from community healthcare centers of Seoul and Busan and had smaller sample size than original GWAS samples, we could not rule out the remaining 17 SNPs for the possible association. The 20 SNPs of GWAS 
Table 4 Effect sizes and p-values of 18 SNPs in replication samples

\begin{tabular}{|c|c|c|c|c|c|c|c|c|c|c|c|c|c|c|c|c|}
\hline \multirow[b]{3}{*}{ CHR } & \multirow[b]{3}{*}{ SNPS } & \multirow[b]{3}{*}{ Linked genes } & \multirow[b]{3}{*}{$M$} & \multicolumn{5}{|c|}{ Replication study $(\mathrm{n}=3703)$} & \multicolumn{8}{|c|}{ Meta analysis } \\
\hline & & & & \multirow[b]{2}{*}{$M A F$} & \multicolumn{2}{|c|}{ Systolic blood pressure } & \multicolumn{2}{|c|}{ Diastolic blood pressure } & \multicolumn{4}{|c|}{ Systolic blood pressure } & \multicolumn{4}{|c|}{ Diastolic blood pressure } \\
\hline & & & & & beta \pm se & P-value & beta \pm se & P-value & BETA & $S B P$ & $Q$ & $\mathrm{P}$ & BETA & $D B P$ & $Q$ & $\mathrm{P}$ \\
\hline 2 & rs295142 & SPATS2L & $\mathrm{C}$ & & & & & & & & & & & & & \\
\hline 2 & rs 1900706 & SPATS2L & $\mathrm{C}$ & 0.186 & $0.32 \pm 0.41$ & $4.4 \times 10^{-1}$ & $0.27 \pm 0.29$ & $3.5 \times 10^{-1}$ & $-0.57 \pm 0.26$ & $2.9 \mathrm{E}-02$ & 6.18 & $1.3 \mathrm{E}-02$ & $-0.40 \pm 0.18$ & $2.5 \mathrm{E}-02$ & 7.47 & $6.3 \mathrm{E}-03$ \\
\hline 2 & rs842825 & SPATS2L & $\mathrm{T}$ & 0.184 & $0.74 \pm 0.42$ & $8.0 \times 10^{-2}$ & $0.17 \pm 0.29$ & $5.7 \times 10^{-1}$ & $-0.38 \pm 0.26$ & $1.5 \mathrm{E}-01$ & 9.64 & $1.9 \mathrm{E}-03$ & $-0.45 \pm 0.18$ & $1.2 \mathrm{E}-02$ & 6.16 & $1.3 \mathrm{E}-02$ \\
\hline 3 & rs3806650 & $B C H E$ & G & 0.238 & $0.54 \pm 0.38$ & $1.6 \times 10^{-} 1$ & $0.55 \pm 0.27$ & $4.0 \times 10^{-1}$ & $-0.43 \pm 0.24$ & $6.6 \mathrm{E}-02$ & 8.87 & $2.9 \mathrm{E}-03$ & $-0.25 \pm 0.16$ & $1.2 \mathrm{E}-01$ & 12.80 & $3.5 \mathrm{E}-04$ \\
\hline 3 & rs11929235 & ILDR1 & A & 0.246 & $-0.30 \pm 0.38$ & $4.4 \times 10^{-1}$ & $0.06 \pm 0.27$ & $8.3 \times 10^{-1}$ & $-0.63 \pm 0.23$ & $6.9 \mathrm{E}-03$ & 1.06 & $3.0 \mathrm{E}-01$ & $-0.36 \pm 0.16$ & $2.3 \mathrm{E}-02$ & 3.53 & $6.0 E-02$ \\
\hline 3 & rs17279465 & SLC6A2O & $\mathrm{C}$ & 0.045 & $-0.04 \pm 0.79$ & $9.6 \times 10^{-1}$ & $-0.87 \pm 0.55$ & $1.2 \times 10^{-1}$ & $-1.35 \pm 0.48$ & $5.0 \mathrm{E}-03$ & 3.85 & $5.0 \mathrm{E}-02$ & $-1.22 \pm 0.33$ & $2.2 \mathrm{E}-04$ & 0.58 & $4.5 \mathrm{E}-01$ \\
\hline 6 & rs9356478 & AL022069 & C & - & - & - & - & - & - & - & - & - & - & - & - & - \\
\hline 6 & rs3789790 & FUCA2 & $G$ & 0.210 & $0.04 \pm 0.40$ & $9.2 \times 10^{-1}$ & $-0.03 \pm 0.28$ & $9.2 \times 10^{-1}$ & $0.63 \pm 0.25$ & $1.1 \mathrm{E}-02$ & 2.96 & $8.5 E-02$ & $0.40 \pm 0.17$ & 1.7E-02 & 3.34 & $6.7 \mathrm{E}-02$ \\
\hline 6 & rs195386 & RNF8 & $\mathrm{T}$ & 0.112 & $-0.37 \pm 0.52$ & $4.7 \times 10^{-1}$ & $-0.38 \pm 0.36$ & $3.0 \times 10^{-1}$ & $-1.14 \pm 0.32$ & $3.9 \mathrm{E}-04$ & 3.03 & $8.2 \mathrm{E}-02$ & $-0.67 \pm 0.22$ & $2.5 \mathrm{E}-03$ & 0.90 & $3.4 \mathrm{E}-01$ \\
\hline 6 & rs6569814 & TAAR3 & $\mathrm{T}$ & 0.327 & $0.44 \pm 0.35$ & $2.1 \times 10^{-1}$ & $-0.11 \pm 0.25$ & $6.6 \times 10^{-1}$ & $0.65 \pm 0.22$ & 2.7E-03 & 0.49 & $4.8 \mathrm{E}-01$ & $0.31 \pm 0.15$ & $3.6 \mathrm{E}-02$ & 4.10 & 4.3E-02 \\
\hline 7 & rs4731112 & RP11-390E23 & $\mathrm{C}$ & 0.197 & $-0.17 \pm 0.41$ & $6.9 \times 10^{-1}$ & $-0.29 \pm 0.29$ & $3.2 \times 10^{-1}$ & $0.65 \pm 0.25$ & $1.0 \mathrm{E}-02$ & 5.43 & $2.0 \mathrm{E}-02$ & $0.32 \pm 0.17$ & $6.2 \mathrm{E}-02$ & 6.39 & $1.1 \mathrm{E}-02$ \\
\hline 7 & rs7357092 & AC006465 & $\mathrm{T}$ & 0.171 & $-0.21 \pm 0.44$ & $6.4 \times 10^{-1}$ & $-0.04 \pm 0.31$ & $9.0 \times 10^{-1}$ & $0.88 \pm 0.27$ & $1.3 \mathrm{E}-03$ & 8.34 & $3.9 \mathrm{E}-03$ & $0.52 \pm 0.19$ & $6.0 \mathrm{E}-03$ & 4.54 & $3.3 \mathrm{E}-02$ \\
\hline 7 & rs3735369 & PUS7 & $\mathrm{T}$ & 0.154 & $0.10 \pm 0.45$ & $8.3 \times 10^{-1}$ & $0.21 \pm 0.32$ & $5.1 \times 10^{-1}$ & $-0.78 \pm 0.28$ & $5.4 \mathrm{E}-03$ & 5.17 & $2.3 \mathrm{E}-02$ & $-0.48 \pm 0.19$ & $1.2 \mathrm{E}-02$ & 6.69 & $9.7 \mathrm{E}-03$ \\
\hline 8 & rs 1786330 & AP001205 & $\mathrm{T}$ & 0.239 & $0.27 \pm 0.39$ & $4.9 \times 10^{-1}$ & $0.14 \pm 0.27$ & $6.0 \times 10^{-1}$ & $-0.53 \pm 0.24$ & $2.5 \mathrm{E}-02$ & 5.94 & $1.5 \mathrm{E}-02$ & $-0.34 \pm 0.16$ & $3.5 \mathrm{E}-02$ & 4.59 & $3.2 \mathrm{E}-02$ \\
\hline 9 & rs3780616 & ALG2 & A & 0.014 & $-4.61 \pm 1.41$ & $1.1 \times 10^{-3}$ & $-1.60 \pm 0.99$ & $1.0 \times 10^{-1}$ & $-3.47 \pm 0.81$ & $2.0 \mathrm{E}-05$ & 0.96 & $3.3 \mathrm{E}-01$ & $-1.73 \pm 0.56$ & $1.9 \mathrm{E}-03$ & 0.03 & 8.7E-01 \\
\hline 11 & rs10768970 & AC068205 & C & 0.232 & $-0.07 \pm 0.39$ & $8.5 \times 10^{-1}$ & $-0.01 \pm 0.27$ & $9.6 \times 10^{-1}$ & $-0.72 \pm 0.24$ & $2.3 \mathrm{E}-03$ & 3.87 & $4.9 \mathrm{E}-02$ & $-0.47 \pm 0.16$ & $3.6 \mathrm{E}-03$ & 4.03 & $4.5 \mathrm{E}-02$ \\
\hline 12 & rs11113470 & ASCL4 & $\mathrm{G}$ & 0.451 & $0.01 \pm 0.32$ & $9.8 \times 10^{-1}$ & $0.00 \pm 0.23$ & $9.9 \times 10^{-1}$ & $0.54 \pm 0.20$ & $8.2 \mathrm{E}-03$ & 3.59 & $5.8 \mathrm{E}-02$ & $0.40 \pm 0.14$ & $4.1 \mathrm{E}-03$ & 4.22 & 4.0E-02 \\
\hline 15 & rs11638762 & AKAP13 & $\mathrm{T}$ & 0.457 & $-0.68 \pm 0.34$ & $4.7 \times 10^{-2}$ & $-0.51 \pm 0.24$ & $3.4 \times 10^{-2}$ & $-0.89 \pm 0.20$ & $1.3 \mathrm{E}-05$ & 0.55 & $4.6 \mathrm{E}-01$ & $-0.48 \pm 0.14$ & $6.3 E-04$ & 0.02 & $8.9 E-01$ \\
\hline 20 & rs4811719 & AL137162 & $\mathrm{T}$ & 0.437 & $-0.08 \pm 0.33$ & $8.1 \times 10^{-1}$ & $0.02 \pm 0.23$ & $9.4 \times 10^{-1}$ & $-0.66 \pm 0.20$ & $1.2 \mathrm{E}-03$ & 4.29 & $3.8 \mathrm{E}-02$ & $-0.36 \pm 0.14$ & $9.6 \mathrm{E}-03$ & 3.82 & $5.1 \mathrm{E}-02$ \\
\hline 22 & rs11089887 & $R P 3-434 P 1$ & $\mathrm{G}$ & 0.185 & $0.11 \pm 0.42$ & $7.9 \times 10^{-1}$ & $0.06 \pm 0.30$ & $8.4 \times 10^{-1}$ & $-0.69 \pm 0.26$ & $7.5 \mathrm{E}-03$ & 5.04 & $2.5 \mathrm{E}-02$ & $-0.45 \pm 0.18$ & $1.2 \mathrm{E}-02$ & 4.22 & $4.0 E-02$ \\
\hline
\end{tabular}

Table 5 Gene function and related diseases

\begin{tabular}{|c|c|c|c|c|c|c|}
\hline $\mathrm{CHR}$ & SNPS & Linked genes & Description & Function & Disease & Reference \\
\hline 2 & $\begin{array}{l}\text { rs295142 } \\
\text { rs1900706 } \\
\text { rs842825 }\end{array}$ & SPATS2L & Spermatogenesis-associated, serine-rich 2-like & - & - & \\
\hline 3 & rs3806650 & $B C H E$ & Butyrylcholinesterase & $\begin{array}{l}\text { Nonspecific cholinesterase found } \\
\text { in the blood plasma }\end{array}$ & Suxamethonium apnea & 24 \\
\hline 3 & rs11929235 & ILDR1 & Immunoglobulin-like domain-containing receptor 1 & - & - & \\
\hline 3 & rs17279465 & SLC6A20 & $\begin{array}{l}\text { Solute carrier family } 6 \text { (proline IMINO transporter), } \\
\text { member } 20\end{array}$ & $\mathrm{Na}+$ and $\mathrm{Cl}$ - coupled transporter & Iminoglycinuria & 25 \\
\hline 6 & rs3789790 & FUCA2 & Fucosidase, alpha-L-2, plasma & Plasma alpha-L-fucosidase & Fucosidosis & 26 \\
\hline 6 & rs195386 & RNF8 & Ring finger protein 8 & $\begin{array}{l}\text { A ubiquitin ligase }(E 3) \text { in the } \\
\text { ubiquitination of certain nuclear proteins. }\end{array}$ & - & 27 \\
\hline 6 & rs6569814 & TAAR3 & Trace amine-associated receptor 3 (gene/pseudogene) & Pseudogene & - & 28 \\
\hline 7 & rs3735369 & PUS7 & Pseudouridylate synthase 7 homolog & - & - & \\
\hline 9 & rs3780616 & $A L G 2$ & $\begin{array}{l}\text { Asparagine-linked glycosylation 2, alpha-1,3- } \\
\text { mannosyltransferase homolog } \\
\text { (Saccharomyces cerevisiae) }\end{array}$ & $\begin{array}{l}\text { Mannosylating Man(2)GlcNAc(2)- } \\
\text { dolichol diphosphate }\end{array}$ & Glycosylation type Ih & 29 \\
\hline 12 & rs11113470 & ASCL4 & Achaete-scute complex homolog 4 & Skin development & - & 30 \\
\hline 15 & rs11638762 & AKAP13 & A kinase (PRKA) anchor protein 13 & $\begin{array}{l}\text { Regulating the Rho/Rac GTPase cycle } \\
\text { (brx in mouse), cardiac development }\end{array}$ & Leukemia, cancer & 21 \\
\hline
\end{tabular}

had the moderate $P$-values $\left(7.8 \times 10^{-3}\right.$ to $\left.9.3 \times 10^{-5}\right)$, and thus they were not considered the candidate region of blood pressure in the previous GWAS study by using the whole SNPs of the Affymetrix SNP chip. ${ }^{8}$ Nevertheless, three SNPs revealed the significant $P$-value and two of them revealed the same effect direction. Therefore, our approach that is the function-oriented association suggests a new solution of highthroughput GWAS.
The rs1 1638762 is the most significant result and located in a kinase anchor protein 13 (AKAP13). AKAP13 binds to the regulatory subunit of protein kinase A, anchoring it to discrete areas within cells. AKAP13 also binds RhoA to activate the Rho family GTPase; ${ }^{17-19}$ the GTPase is a mediator of cardiac hypertrophy, and AKAP13 is upregulated in hypertrophic cardiomyocytes. ${ }^{20}$ Moreover, cardiomyocytes in AKAP13-null mice have impaired sarcomere formation, causing 


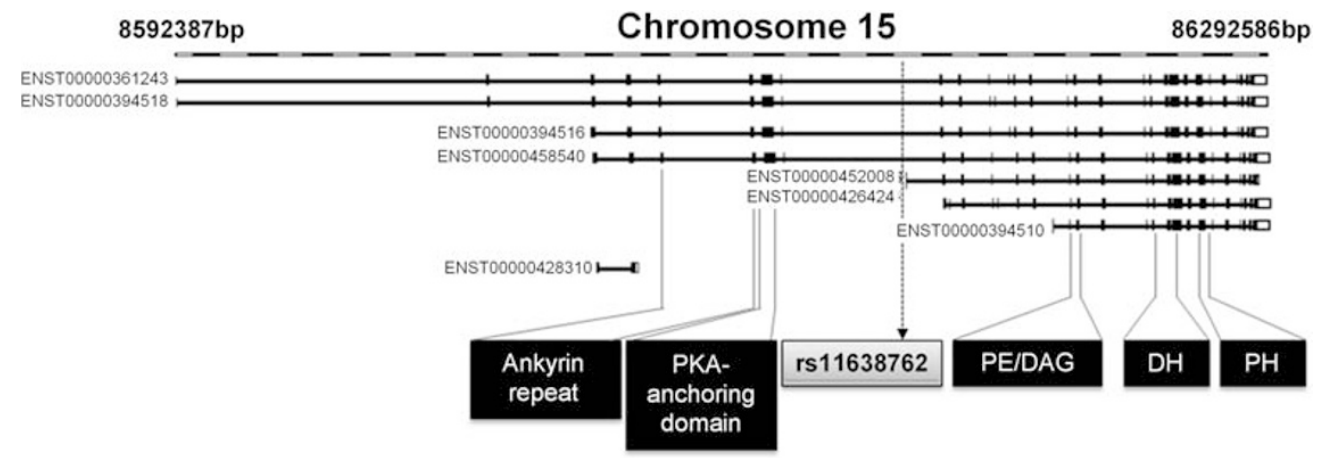

Figure 2 Genomic location, transcript variants and functional domain positions of the AKAP13 gene obtained from Ensembl (http://www.ensembl.org/ index.html). PE/DAG: protein kinase C-like diacyglycerol binding site (PE/DAG), DH: dbl oncogene homology, PH: pleckstrin homology. rs11638762 is a significantly associated SNP -2682 bp of ENST00000452008 (bold highlighted).

embryos to develop thin-walled hearts and die at E10.5-11.0. ${ }^{21}$ Altered AKAP13 expression influences genes that are essential for normal cardiac development, particularly myocyte enhancer factor $2 \mathrm{C}^{21}$ Therefore, we speculate that rs11638762, an SNP of AKAP13, alters AKAP13 expression levels, leading to variations in wall thickness in the heart, which might influence blood pressure, secondary to the pumping capacity of the heart.

AKAP13 lies on chromosome 15q24-25 (ref. 22) and has eight transcriptional variants, generated by alternative splicing, as observed in Ensembl (http://www.ensembl.org/index.html) (Figure 2). The longest transcript contains at least five domains, such as an ankyrinrepeat domain, a PKA-anchoring domain, a protein kinase C-like diacyglycerol consensus binding site, a dbl oncogene homology domain, and a pleckstrin homology domain; a dbl oncogene homology-pleckstrin homology domain acts as a guanine nucleotide exchange factor for the Rho GTPase. ${ }^{17}$

rs11638762 is located upstream of three AKAP13 transcripts (ENST00000452008, ENST00000426424 and ENST00000394510), 2682 bp upstream of ENST00000452008 (Figure 2). However, the SNP located on intron of the other AKAP13 transcript. All these three transcripts contain dbl oncogene homology-pleckstrin homology domains that bind to Rho. Moreover, the minor allele of this SNP was predicted by the TFsearch program to disrupt GATA-3 binding to the AKAP13 promoter. $^{23}$ The major allele (A) is the GATA-3 binding site is NNGATARNG (TF score $=94.7$ ); the underlined nucleotide is changed in the SNP (rs11638762) to T, possibly interrupting its transcriptional regulation. However, we should mention that if three transcripts are not the major transcripts of AKAP13, the effect of rs11638762 on differential expression of AKAP13 might not be influential on the regulation of blood pressure.

The rs3780616 revealed consistent association for SBP, but not DBP. The SNP located on the upstream of asparagines-linked glycosylation 2 alpha-1,3-mannosyltransferase homolog (ALG2). ${ }^{29}$ The ALG2 gene has three alternative transcripts, and the SNP locates at $-3522 \mathrm{bp}$ upstream of all three transcripts. The minor allele of this SNP was predicted by the TF search program to create a new cAMP response element-binding (CREB) to the ALG2 promoter. The minor allele (T) sequence of the SNP site can be change to CREB binding site TGACCTCA (TF score $=86.6$ ), possibly induce the CREB binding. It has been known that ALG2 is related to cell cycle, ${ }^{31}$ but there is no report related to blood pressure regulation in ALG2 gene.

In conclusion, rs11638762, located upstream of the AKAP13 gene, has a robust association with blood pressure, and it lies in a GATA-3 binding site, rendering the SNP a potential cause of blood pressure variations through alterations in AKAP13 expression levels. This study also demonstrates that our approach of using functional SNPs, such as regulatory SNPs (TFBS-SNPs) in addition to a previous GWAS on nonsynonymous SNPs, ${ }^{9}$ identifies the genetic variations that biologically linked to blood pressure regulation.

\section{CONFLICT OF INTEREST}

The authors declare no conflict of interest.

\section{ACKNOWLEDGEMENTS}

This work was supported by a grant from the Kyung Hee University in 2010 (KHU-20100133). The authors would like to thank the Korean National Institute of Health, which contributed to the collection and phenotypic characterization of the clinical samples and the genotyping and analysis of the GWA data.

1 Fields, L. E., Burt, V. L., Cutler, J. A., Hughes, J., Roccella, E. J. \& Sorlie, P. The burden of adult hypertension in the United States 1999 to 2000: a rising tide. Hypertension 44, 398-404 (2004).

2 Lawes, C. M., Vander Hoorn, S. \& Rodgers, A. Global burden of blood-pressure-related disease, 2001. Lancet 371, 1513-1518 (2008).

3 WTCCC. Genome-wide association study of 14000 cases of seven common diseases and 3000 shared controls. Nature 447, 661-678 (2007).

4 Frazer, K. A., Murray, S. S., Schork, N. J. \& Topol, E. J. Human genetic variation and its contribution to complex traits. Nat. Rev. Genet. 10, 241-251 (2009).

5 Mohlke, K. L., Boehnke, M. \& Abecasis, G. R. Metabolic and cardiovascular traits: an abundance of recently identified common genetic variants. Hum. Mol. Genet. 17, R102-108 (2008).

6 Newton-Cheh, C., Johnson, T., Gateva, V., Tobin, M. D., Bochud, M., Coin, L. et al. Genome-wide association study identifies eight loci associated with blood pressure. Nat. Genet. 41, 666-676 (2009).

7 Levy, D., Ehret, G. B., Rice, K., Verwoert, G. C., Launer, L. J., Dehghan, A. et al. Genome-wide association study of blood pressure and hypertension. Nat. Genet. 41, 677-687 (2009).

8 Cho, Y. S., Go, M. J., Kim, Y. J., Heo, J. Y., Oh, J. H., Ban, H. J. et al. A large-scale genome-wide association study of Asian populations uncovers genetic factors influencing eight quantitative traits. Nat. Genet. 41, 527-534 (2009).

9 Hong, K. W., Jin, H. S., Lim, J. E., Cho, Y. S., Go, M. J. et al. Non-synonymous singlenucleotide polymorphisms associated with blood pressure and hypertension. J. Hum Hypertens. 24 (11), 763-774 (2010).

$10 \mathrm{Wu}$, L. C., Horng, J. T. \& Chen, Y. A. A computation to integrate the analysis of genetic variations occurring within regulatory elements and their possible effects. J. Comput. Biol. 16, 1731-1747 (2009).

11 Kim, H. A., Chun, H. Y., Kim, S. H., Park, H. S. \& Suh, C. H. C-reactive protein gene polymorphisms in disease susceptibility and clinical manifestations of Korean systemic lupus erythematosus. J. Rheumatol. 36, 2238-2243 (2009).

12 Kawai, T., Takeshita, S., Imoto, Y., Matsumoto, Y., Sakashita, M., Suzuki, D. et al. Associations between decay-accelerating factor polymorphisms and allergic respiratory diseases. Clin. Exp. Allergy 39, 1508-1514 (2009).

13 Egli, R. J., Southam, L., Wilkins, J. M., Lorenzen, I., Pombo-Suarez, M., Gonzalez, A et al. Functional analysis of the osteoarthritis susceptibility-associated GDF5 regulatory polymorphism. Arthritis Rheum. 60, 2055-2064 (2009). 
14 Harendza, S., Stahl, R. A. \& Schneider, A. The transcriptional regulation of podocin (NPHS2) by Lmx1b and a promoter single nucleotide polymorphism. Cell Mol. Biol. Lett. 14, 679-691 (2009).

15 Rabbee, N. \& Speed, T. P. A genotype calling algorithm for affymetrix SNP arrays. Bioinformatics 22, 7-12 (2006).

16 Purcell, S., Neale, B., Todd-Brown, K., Thomas, L., Ferreira, M. A., Bender, D. et al. PLINK: a tool set for whole-genome association and population-based linkage analyses. Am. J. Hum. Genet. 81, 559-575 (2007).

17 Diviani, D., Soderling, J. \& Scott, J. D. AKAP-Lbc anchors protein kinase A and nucleates Galpha 12-selective Rho-mediated stress fiber formation. J. Biol. Chem. 276, 44247-44257 (2001).

18 Schmidt, A. \& Hall, A. Guanine nucleotide exchange factors for Rho GTPases: turning on the switch. Genes Dev. 16, 1587-1609 (2002).

19 Sterpetti, P., Hack, A. A., Bashar, M. P., Park, B., Cheng, S. D., Knoll, J. H. et al. Activation of the Lbc Rho exchange factor proto-oncogene by truncation of an extended $C$ terminus that regulates transformation and targeting. Mol. Cell Biol. 19, 1334-1345 (1999).

20 Carnegie, G. K., Soughayer, J., Smith, F. D., Pedroja, B. S., Zhang, F., Diviani, D. et al. AKAP-Lbc mobilizes a cardiac hypertrophy signaling pathway. Mol. Cell 32, 169-179 (2008).

21 Mayers, C. M., Wadell, J., McLean, K., Venere, M., Malik, M., Shibata, T. et al. The RHO-guanine nucleotide exchange factor AKAP13 (BRX) is essential for cardiac development in mice. J. Biol. Chem. 285, 12344-12354 (2010).

22 Miller, B. T., Rubino, D. M., Driggers, P. H., Haddad, B., Cisar, M., Gray, K. et al. Expression of brx proto-oncogene in normal ovary and in epithelial ovarian neoplasms. Am. J. Obstet. Gynecol. 182, 286-295 (2000).

23 Ohneda, K. \& Yamamoto, M. Roles of hematopoietic transcription factors GATA-1 and GATA-2 in the development of red blood cell lineage. Acta. Haematol. 108, 237-425 (2002).
24 Barta, C., Sasvari-Szekely, M., Devai, A., Kovacs, E., Staub, M. \& Enyedi, P. Analysis of mutations in the plasma cholinesterase gene of patients with a history of prolonged neuromuscular block during anesthesia. Mol. Genet. Metab. 74, 484-488 (2001).

25 Broer, A., Balkrishna, S., Kottra, G., Davis, S., Oakley, A. \& Broer, S. Sodium translocation by the iminoglycinuria associated imino transporter (SLC6A20). Mol. Membr. Biol. 26, 333-346 (2009).

26 O'Brien, J. S., Willems, P. J., Fukushima, H., de Wet, J. R., Darby, J. K., Di Cioccio, R. et al. Molecular biology of the alpha-L-fucosidase gene and fucosidosis. Enzyme 38, 45-53 (1987).

27 Ishikawa, K., Nagase, T., Suyama, M., Miyajima, N., Tanaka, A., Kotani, H. et al. Prediction of the coding sequences of unidentified human genes. $X$. The complete sequences of 100 new cDNA clones from brain which can code for large proteins in vitro. DNA Res. 5, 169-176 (1998).

28 Lindemann, L., Ebeling, M., Kratochwil, N. A., Bunzow, J. R., Grandy, D. K. \& Hoener, M. C. Trace amine-associated receptors form structurally and functionally distinct subfamilies of novel G protein-coupled receptors. Genomics 85, 372-385 (2005).

29 Thiel, C., Schwarz, M., Peng, J., Grzmil, M., Hasilik, M., Braulke, T. et al. A new type of congenital disorders of glycosylation (CDG-li) provides new insights into the early steps of dolichol-linked oligosaccharide biosynthesis. J. Biol. Chem. 278, 22498-22505 (2003).

30 Jonsson, M., Bjorntorp Mark, E., Brantsing, C., Brandner, J. M., Lindahl, A. \& Asp, J. Hash4, a novel human achaete-scute homologue found in fetal skin. Genomics 84, 859-866 (2004).

31 Hoj, B. R., la Cour, J. M., Mollerup, J. \& Berchtold, M. W. ALG-2 knockdown in HeLa cells results in G2/M cell cycle phase accumulation and cell death. Biochem. Biophys. Res. Commun. 378, 145-148 (2009). 